\title{
Design and Implementation of IoT Project Reader
}

\author{
Yang Feng \\ School of Physics and Mechanical \& Electrical \\ Engineering \\ Hechi University \\ Yizhou, Guangxi, China \\ oyfo@163.com
}

\author{
Lan Chun-liang* \\ Hechi University \\ Yizhou, Guangxi, China \\ 1c17413@163.com \\ *Corresponding author
}

\author{
Liu Di \\ School of Physics and Mechanical \& Electrical \\ Engineering \\ Hechi University \\ Yizhou, Guangxi, China \\ liudi611@126.com \\ Song Hua-ning \\ School of Physics and Mechanical \& Electrical \\ Engineering \\ Hechi University \\ Yizhou, Guangxi, China \\ shn_2008@163.com
}

\section{OVERALL DIAGRAM OF THE FORMALIZATION DEPLOYS SYSTEM}

\begin{abstract}
To meet the project' requirements of IoT application systems, we designed a $\mathrm{UHF}$ reader that based on the STM32F103ZET6 and RMU900+. The reader can work in three modes which are local control mode, standalone network control mode and centralized network control mode. While working in the network mode, single information terminal can simultaneously control and monitor 16 readers. With this system, we can facilitate the overall system deployment and dynamic debugging, it also can improve the performance of system and shorten the construction cycle.
\end{abstract}

Keywords- RFID reader; Reader networking; RMU900+; Power adapting;IoT application system

\section{INTRODUCTION}

In the formalization IoT application system project deployment process, the RFID reader operational parameter adjustment is an extremely important link. The technical state of the link will affect the system realization and deployment effectiveness of work. The widespread adoption of the construction method is a single read-write, a case-by-case basis, and debugging is not taking into account the multi-read, coordination and management of the problem, the size of the system reaches a certain level, the read and write-to-server interaction and interference makes deployment becomes more difficult ${ }^{[1]}$. The present situation has affected the formalization system construction efficiency and the work effect, hindered the networking application system scale further forward to develop. In this paper, the author based on the STM32F103ZET6 kernel and RMU900+ RF module, designed and implemented a IoT reader project, and cooperate with all the existing information terminal (the gathering node), and complete the whole system of project deployment and testing. We may achieve the system formalization fast deployment and the common control by using the system.
The whole system is shown in Fig. 1, it consists of the main control machine, the Transmission network, the Information terminal and the IoT reader project. The main control machine can choose any ordinary PC machine can run Windows system; the transmission network based on the actual environment and the scale of deployment and reliability requirements, it can choose one from LAN, WIFI, and $3 \mathrm{G}$ network; the information terminal uses its own multiple communication channel to connect all kinds of reader project to the transmission network. The system is based on the IoT multi-source information terminal which based on $\mathrm{S} 3 \mathrm{C} 6410^{[2]}$; the reader project of IoT is for this design. In the overall system operation process, we can long-distance register the information terminal by the main control computer, and set working parameter device of several readers dynamic.

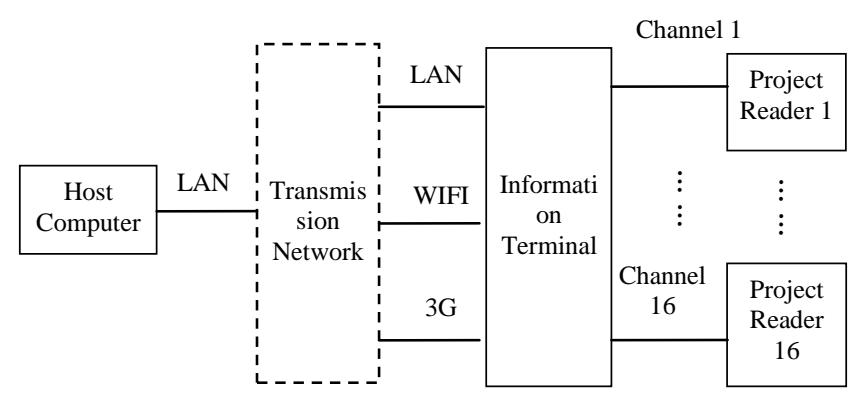

Figure 1. Overall block diagram of the system

\section{THE HARDWARE DESIGN OF INTERNET ENGINEERING READER}

Based on STM32 and RMU900+ thing networking project reader hardware diagram as shown in Fig. 2, STM32F103ZET6 MCU is the systems control and the corre-spondence core, peripheral local controls the pressed 
key, the liquid crystal display module, for its disposition carries on the correspondence with the gathering node and the RMU900+ module the serial interface and so on; The RMU900+ radio frequency module uses the SMA connection and the radio frequency antenna connected and through the serial mode and the MCU correspondence ${ }^{[3,4]}$.

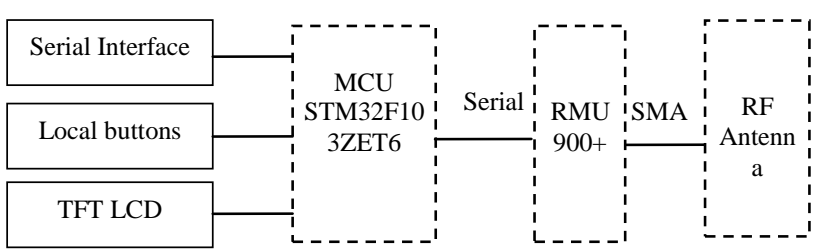

Figure 2. Project reader hardware block

\section{A. MCU selection}

Taking into overall account performance, the cost, the power consumption, the reliability, and other factors, we select ARM Cortex-M3 core STM 32F103ZET 6 as the system MCU, the MCU is a 32-bit architecture, with the LQFP - 144 package, 2.0 - $3.6 \mathrm{~V}$ power, the main frequency $72 \mathrm{MHz}$, with $512 \mathrm{~K}$ chip FLASH and $64 \mathrm{~K}$ chip RAM and to support in-circuit programming the IAP, support for up to 80 IO (TTL level), with 2-way I2C interface and 5-way USART interface and a USB from device interface. All kinds of resources to meet the system requirements, and there is a certain degree of scalability.

\section{B. Radio frequency processing module}

According to park vehicles early-warning system's read and write distance, lane, vehicle identification of needs, we select the UHF band UHF read and write module RMU $900+$. The module is UHF RFID read-write, the core components and integrated the PLL, launch, receiving, couplers, and MCU components such as, the working voltage of $3.3 \mathrm{~V}$ by UART interface with the end device to communicate, the module with its own $\mathrm{C}$ language API library, it will be possible to significantly reduce development cycles and reduce risk, post-can also run in accordance with the relevant function to modify. The module core circuitry is shown in Fig. 3.

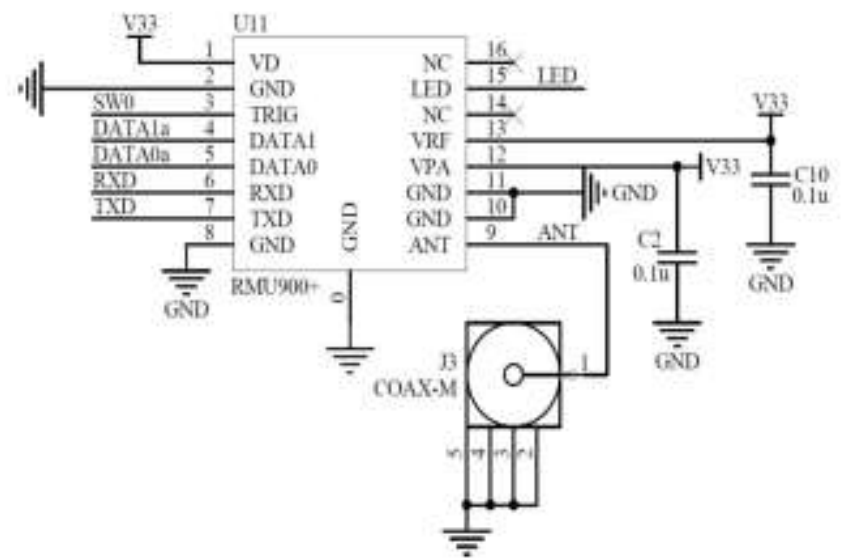

Figure 3. The principle diagram of the radio frequency processing module

The module practical work frequency may according to have to have custom-made in the $840 \sim 960 \mathrm{MHz}$ scope, may support EPC C1 GEN2 and the ISO18000-6C agreement, the peak power output may reach $30 \mathrm{dBm}$, necessary $13 \mathrm{dBi}$ increases the radio frequency antenna, the system output may reach $1 \mathrm{~W}$, may achieve $5 \mathrm{~m}$ to the compatible ISO18000-6C agreement electronic label the read-write distance.

\section{Antenna matching and selection}

This system is equipped with RFID tags is compatible with ISO 18000-6c protocol of passive electronic tags, tags within the transmission circuit to work the amount of energy needed to antenna by radiation in the form of providing by reading and writing, have higher request for the technical parameters of antenna. According to radio frequency radiation power calculation principle ${ }^{[5]}$, assuming that the antenna ports standing wave ratio is 1.5 , according to the formula $S=10 \log$ [pattern (VSWR 1)/pattern (VSWR + 1)], $\mathrm{S}=-7 \mathrm{~dB}$, can be obtained if set to read and write device transmitted power of $30 \mathrm{dBm}(1 \mathrm{w})$, then the radiation to the label of receiving circuit for 23 $\mathrm{dBm}$ signal strength, the signal will be into together with the antenna receives the useful signal at the receiving end, and $23 \mathrm{dBm}$ strong signal mixer after attenuation will just reached the receiving end of input $1 \mathrm{~dB}$ compression point. If legibility antenna ports of standing wave ratio greater than 1.5 , the reflected signal strength will be higher than that of $23 \mathrm{dBm}$, namely more than mixer $1 \mathrm{~dB}$ compression point, leading to a receiver circuit block. Therefore, when selecting a read/write device antenna, ask its standing wave ratio is not greater than 1.5 , to ensure that in the case of the output power is enough receiver can work normally. In addition, RMU900 + module for antenna port impedance matching for $50 \mathrm{ohm}$, table 1 lists the different transmission power, measured by the antennas with different gain actual card read distance (ISO $18000-6 \mathrm{c}$ white card) ${ }^{[6,7]}$.

\section{Buttons and display module}

In order to improve the system's ease of use in standalone mode, we have set up five keys, respectively is DOWN, the SELECT, LEFT, RIGHT, UP, pull UP resistance of each key-press through external $10 \mathrm{k}$ is linked with the I/O port of MCU, and, key circuit as shown in fig. 4.

Local display part adopts the 3.0 inch TFT LCD screen, its resolution is WQVGA $(400 \times 240)$, screen directly connected through the I/O port with MCU. Hardware system objects is shown in fig. 5 . 


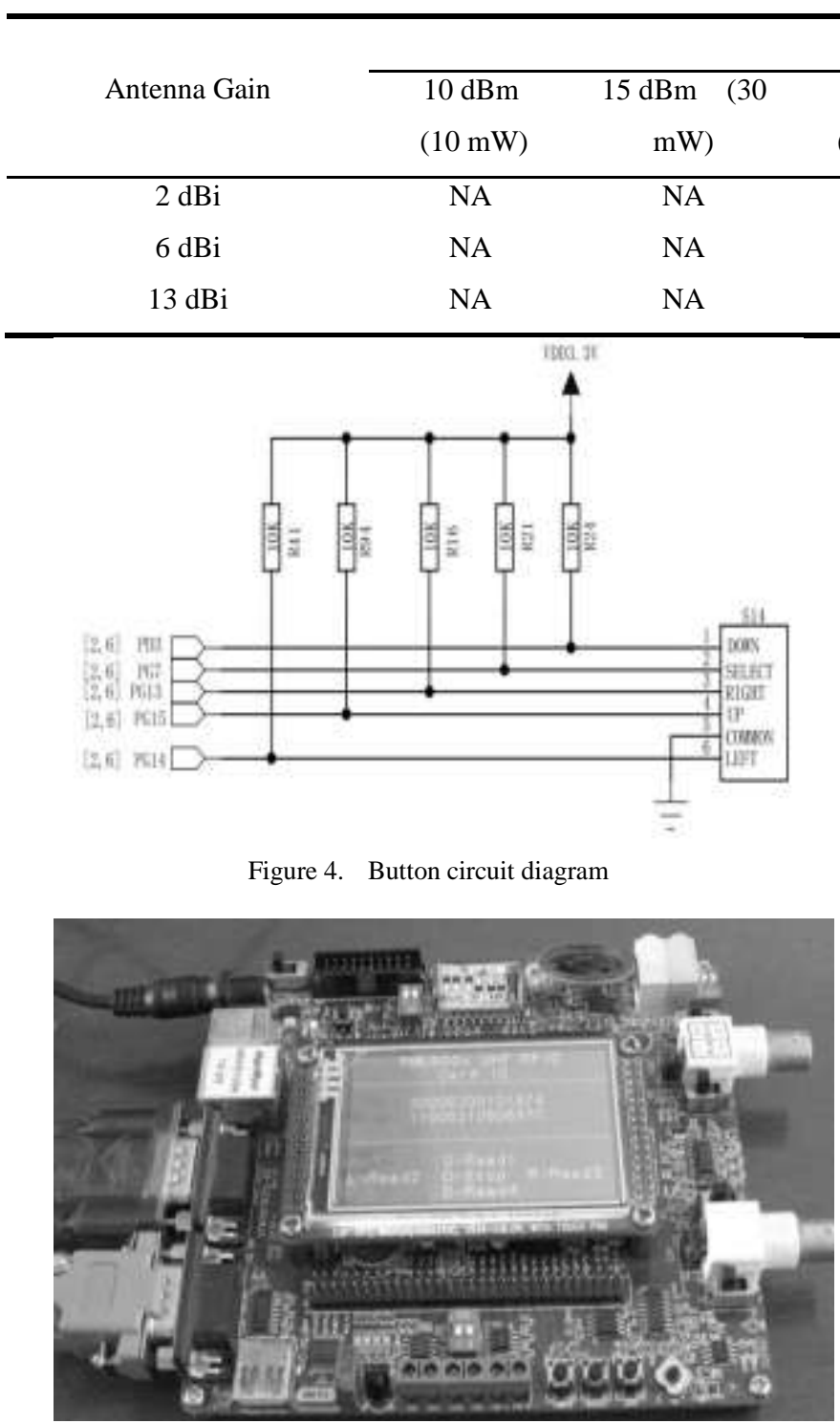

Figure 5. Device realy picture

\section{SYSTEM SOFTWARE DESIGN}

\section{A. development environment}

Read device working procedure's development and is written in the Real View MDK embedded in the Keil $\mathrm{uVision} 4$, program design according to the function module into separate files, separate debugging success, then function integration and compiler generated. Hex file, through the downloader to download into the flash of $\mathrm{MCU}^{[8-10]}$. Work under the control mode of main control machine using VC to write software, running on Windows $\mathrm{PC}$ to run normally, reduce the system requirements for hardware, at the same time improve flexibility .

\section{B. The communication parameters and data packet format}

The RMU900+ module carries on the correspondence through UART and MCU, MCU needs to defer to the
Output Power

$\begin{array}{ccccc}20 \mathrm{dBm} & 23 \mathrm{dBm} & 27 \mathrm{dBm} & (0.5 & 30 \mathrm{dBm} \\ 100 \mathrm{~mW}) & (200 \mathrm{~mW}) & \mathrm{W}) & (1 \mathrm{~W}) \\ 0.45 \mathrm{~m} & 0.5 \mathrm{~m} & 0.5 \mathrm{~m} & 0.5 \mathrm{~m} \\ \mathrm{NA} & 2 \mathrm{~m} & >2 \mathrm{~m} & >2 \mathrm{~m} \\ 4.5 \mathrm{~m} & 5 \mathrm{~m} & >5 \mathrm{~m} & >5 \mathrm{~m}\end{array}$

stipulation the data format to order and to receive its returns to the RMU900+ transmission the information. The correspondence baud rate is the $57600 \mathrm{bps}, 8$ bit data position, 1 stop position, non-parity check.

The data packets that MCU sent to RMU 900+ module is called the "command" and RMU 900+ module return to MCU packets called "response". The format of data packets is shown in Table 2 and table 3 .

\section{Program implementation process and the test}

Main program execution flow is shown in figure 6 , the first electric reset MCU initialization, and then reset RMU900+ module, so can select the card read command buttons and sent via a serial port to the RMU900+ module. If execution is card read command, the reader sends the card data to the serial port of MCU, the main program for data processing in the interrupt function, and the ID number in the array, then the call display function will card number information displayed on the LCD, the run ends and waits for the next command.

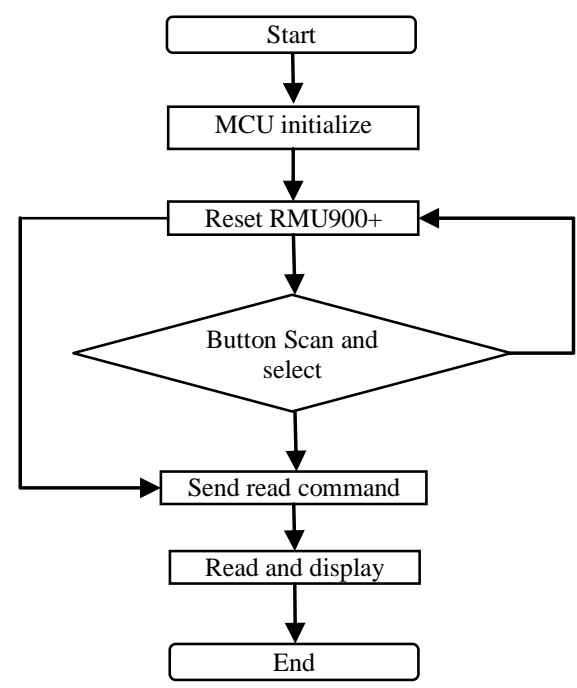

Figure 6. The main program flow chart 


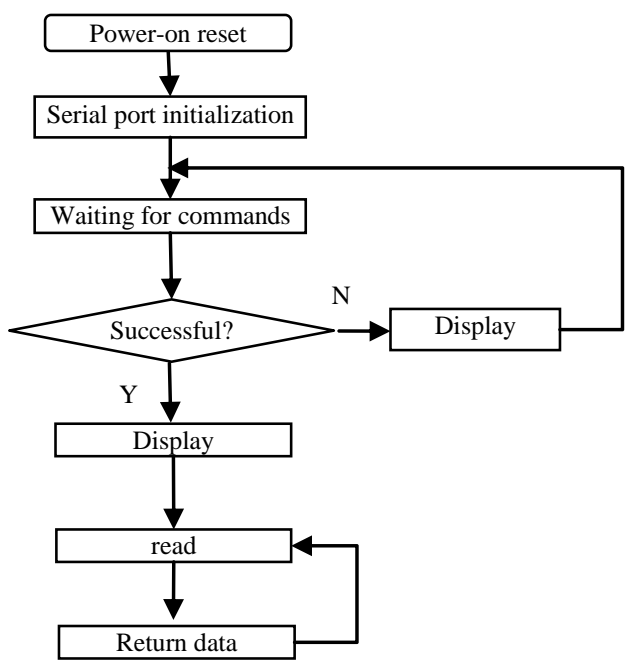

Figure 7. RMU900+ internal program flow

Internal RMU900+ module has been integrated with ISO 18000-6c protocol, read device used in ISO 18000$6 \mathrm{c}$ protocol select, inventory, and access to the three basic operations to manage the label, each operation is composed of a number of commands. Because the module has memory function, if not to electric reset or send stop command operation, the module will have been performing for the first time that received the command mode to read card. The module internal process is shown in fig. 7.

System centralized testing interface as shown in fig. 8 , in the control interface choice to control the reading and writing, and then click connect button to set working parameters of the read device from the network.Card ID number can be displayed in the left side if it is in the process of running the read command. It can also choose to "all channel" that I can realize all read and write device set centrally in channel selection drop-down list, and ID information on the left shows in $\operatorname{turn}^{[11,12]}$.

\section{CONCLUSION}

In this paper, the author uses STM32F103ZET6 and RMU900+ uhf RFID read mod-ule was designed and implemented the Internet of things engineering RFID reading and writing. Complete a trial production of the hardware and software development and commissioning, the running results show that the read device can reach the Inter-net of things environment zone of early warning management of vehicle requirements.in these three kinds of operating modes including including the local control, single machine control and centralized network control. Compared with existing business, speaking, reading and writing, this equipment has a relatively unique technical features and practical value, the overall system controllability is stronger.

\section{ACKNOWLEDGMENT}

This project was supported by Research projects of hechi university (2013ZX-N004; 2013A-N004)

\section{REFERENCES}

[1] Wang Xiao. "Adjacent channel interference" problem solution for RFID applications. J. China Auto-ID.2007, 02:46-47+30

[2] Liu Di, Zhang Xiang-li. IOT'S Multi-source information terminal based on S3C6410. J. Journal of Hechi University. 2012,05:92-97.

[3] Liu Jun-jun. Design of UHF RFID Reader. D. Nanjing University of Posts and Telecommunications, 2011.

[4] Liu Huai-da, Tan Jie, Yang Yi-ping. Design and implementation of security reader. J. Application of Electronic Technique. 2009, 05:120-122+125

[5] Xue Qing-jun, Han Jin. Achieving technique of multitask bas ed on Keil C51 embedded operation system. J. Microcomputer Information, 2007, 05:63-64+77.

[6] Jay Singh, Navjit Brar, and Carmen Fong. The State of RFID Applications in libraries.Information Technology and Libraries. 2006.3:24-32.

[7] Ngai, E.W.T.\&Karen K.L.Moon\&Frederick J. Riggins\&Candace Y. Yi.RFID research:An academic literature review(1995-2005)and future research directions[J].International Journal of Production Economics,2006,12:1-11.

[8] Information Technology-Radio Frequency Identification for Item Management Part 6: Parameters for Air Interface Communication at 860-960MHz, 1800-6 ISO/IEC 2006.

[9] Guido Bertoni,Luca Breveglieri,Matteo Venturi.ECC Hardware Coprocessors for 8-bit Systems and Power Consumption Considerations[J].The IEEE Computer Society,2006.

[10] Ciaran J.Mcivor,Maire Mcloone,John V.Mccanny. Hardware Elliptic Curve Cryptographic Processor over GF(p)[J].Inclusion in a Future Issue,2006(6):87-95.

[11] HU Zhi-xi.Design of a UHF reader in RFID production linesystem [J]. Microcomputer Information, 2009(05)25-27.

[12] TIAN Jing;CHU Haibing.Design of RFID ReaderWriter[J]. Modern Electronics Technique. 2009(01):17-19. 